\title{
Managing aircraft mobility in a context of the ATN/IPS network
}

\author{
Tran Nguyen Alexandre \\ Telecom/ReSCo \\ ENAC \\ Toulouse, France \\ hoang.alexandre.tran.nguyen@ rockwellcollins.com
}

\author{
Pirovano Alain \\ Telecom/ReSCo \\ ENAC \\ Toulouse, France \\ alain.pirovano@enac.fr
}

\author{
Larrieu Nicolas \\ Telecom/ReSCo \\ ENAC \\ Toulouse, France \\ nicolas.larrieu@enac.fr
}

\begin{abstract}
For the sake of Air Traffic Management modernization, civil aviation organizations are currently developing IPS for Aeronautical Safety Services in the new ATN/IPS infrastructure. This includes to define new airborne and ground- based communication systems capable of managing both air traffic services (ATS) and aeronautical operational communications (AOC) safety services. One of the main challenges in this new ATN/IPS network is the IPv6 mobility problem. This paper proposes a solution which takes both advantages of ground based Locator/Identifier Separation Protocol and Proxy Mobile IPv6 to manage all the aircraft mobility scenarios. A dedicated OMNeT++ simulation model is also provided and shows the performances of our solution.
\end{abstract}

Index Terms-ATN/IPS, Mobility, LISP, PMIPv6, OMNeT++

\section{INTRODUCTION}

In the future Air Traffic Management (ATM) concept, in order to increase safety, airspace capacity, flight and cost efficiency, applications will need to exchange more and more data with the aircraft. Aeronautical Telecommunication Network over IP (ATN/IPS) will be the new standard of the future ATM infrastructure. It will allow a more efficient use of datalink communications, which will become in the next decades a major mean of communication. This Future Communication Infrastructure ( $\mathrm{FCI}$ ) aims at providing a resilient and secure way to transfer flight critical data and voice communications over digital links. To meet this requirement, new functionalities in terms of mobility, security and Quality of Services (QoS) must be defined due to the particularities of the aeronautical environment.

This paper focuses on the problem of network mobility in the FCI. The International Civil Aviation Organization (ICAO) is currently working on this aspect but has not defined yet the solutions. Proposals have been made such as GroundLocator/Identifier Separation Protocol (G-LISP) [1], Proxy Mobile IPv6 (PMIPv6) [2] and Asymmetric Extended Route Optimization (AERO) [3]. All these solutions resolve only partial mobility issues. PMIPv6 and AERO are intra-domain mobility solutions, whereas Ground-LISP defines a solution to cope with the inter-domain mobility issue. The mobility solution also has to take into consideration multilink properties, which are required by the operating concept defined in SESAR 15.2.4.
The aim of this paper is to deal with both mobility scenarios, which have been identified by T.Whyman in the ICAO mobility working sub-group as use case scenarios. Particularly, coupling G-LISP with PMIPv6 may be an adequate solution as it will allow to solve intra-domain and inter-domain aircraft mobility. This paper will first present the architecture of the ATN/IPS network and all the entities that play a role in the mobility solution. After a short description of PMIPv6 and LISP, the paper describes how these protocols can be combine to handle the aircraft network mobility. A dedicated simulation model we have developed under the OMNeT++ software is provided and a performance evaluation and analysis of our proposal is performed.

\section{ATN/IPS OVERVIEW}

The Future Communication Infrastructure (FCI) designed for ATN/IPS will be composed of different subnetworks:

- An aircraft which is considered as a mobile subnetwork hosting airborne end systems (A-E). It can be seen either as a mobile node or a mobile network at the IP level. It includes an airborne router (A-R) responsible for the link selection and the management of the inter-technology handoffs, and also airborne radios (AR) providing the communication over air/ground links.

- Access Networks, which refer to the subnetwork providing the air/ground link between the aircraft and the ground systems. They include specific radio communication means to reach the aircraft (such as VDLm2, LDACS, SATCOM, and future communication means), an Access Ground router (AC-R) which is the last hop router towards the aircraft, and an Air/Ground router (A/G-R) to connect the access network to the ATN/IPS ground network. Access networks belong to Communication Service Providers (CSP), whose aim is to provide a connectivity to the aircraft. Hence, several access networks may be grouped into a same administrative domain.

- Applicative Service Providers are ground end-systems communicating with airborne end-systems ensuring a certain service. Some examples of Applicative Service Provider are ATC Centre and ANSP whose role are to 
manage the air traffic, or AOC centre which are dedicated to airlines operations.

- Ground ATN/IPS Internetwork is the core network of ATN/IPS. It maintains a connection between all the different subnetworks.

The FCI will be the interconnection of all these subnetworks and hence securing an IP end-to-end communication between all the aeronautical stakeholders. The FCI is represented in the Figure 1, in which we illustrate the fact that there should be more than one CSP providing one type of link technology.

Given ATN/IPS network topology, there are 2 mobility problems to solve: intra-domain mobility, where the aircraft stays in the same domain, and inter-domain mobility where the aircraft changes its network access provider. These mobility scenarios can be either horizontal or vertical, meaning that they do not depend on whether the aircraft keeps using the same technology access or changes to another one. Indeed, a CSP may provide more than one access networks to the aircraft via either a terrestrial or a satellite based access technology, so even if the aircraft has to change between communication means, it may remain in the same administrative domain (CSP domain). The main challenge in these mobility scenarios is to provide a seamless handoff to the application layer. This is realized by keeping the same address for the aircraft, so that it does not have to break any application sessions while changing its network access point. To cope with the heterogeneity of the FCI and handle these mobility scenarios efficiently, subdividing the problem into a local mobility scenario and a global mobility scenario will allow to define a scalable and optimized solution. This solution has to take into account the specificities of the aeronautical environment, thats to say, it must minimize the message exchange on the radio link.

\section{MoBility SOLUTION}

\section{A. PMIPv6: an intra-domain mobility solution}

In the first time, we focus on the intra-domain mobility scenario. Instead of relying on a routing protocol (for instance OSPF is proposed in [1]) which has not been developed for such a scenario, using a dedicated mobility protocol is more advantageous. As mentioned in [4], Proxy Mobile IPv6 (PMIPv6) is the first IPv6 network mobility protocol to propose such a solution. It provides indeed a unique address to the aircraft when it enters in the domain. Such feature is not provided by other routing protocols, which only constructs a route to reach the aircraft. It is also compatible with multilink scenarios, making him a good candidate to solve the intradomain handoff.

PMIPv6 has gained much attention in the network \& telecommunication area recently and has been standardized by IETF in RFC 5213 [5]. It is a network-based mobility management protocol, inspired from the Mobile IPv6 protocol. It allows the node mobility management inside an administrative domain, also called a proxy domain, and thus hiding the intradomain node mobility to the rest of the network.

PMIPv6 defines two new entities: a local anchor point (LMA) and a proxy router (MAG). The LMA has the role of the Home Agent inside the domain, so it keeps an entry for each MN inside the proxy domain. This entry contains the Home Network prefix allocated for this MN and its proxy Care-of-Address which is the address of the router the $\mathrm{MN}$ is connected to. While the MAG handles the mobility signaling for any mobiles nodes entering the proxy domain. The handoff between two MAGs belonging to the same proxy domain is described in Figure 2. The previous MAG (pMAG) sends a Binding Deregistration to the LMA so it can erase the pMAG address in the cache. When the nMAG detects a new mobile node by receiving a RS message, it starts the PMIP procedure for the $\mathrm{MN}$ by sending a Binding Update message towards the LMA. The latter modifies the proxy Care-of-Address, i.e the MAG address, in the corresponding entry and replies with a Binding Acknowledgement, containing the home network prefix (HNP) allocated for the MN. The MAG will then send a RA to the MN containing the HNP. The MN will thus receive the same prefix and will still use the corresponding address to communicate. The extension defined in rfc 7864 allows the use of multiple MAGs at the same time to forward packets to the MN.

Without involving the $\mathrm{MN}$ in the mobility signalling exchange, PMIPv6 succeeds to manage its mobility. Moreover, the main advantage of this protocol is to allow a fast handoff between different communication means belonging to the same administrative domain. For instance, suppose that a CSP proposes more than one communication means to the aircraft in some regions (i.e LDACs, and future L2 system), a vertical handoff between these 2 communication means will only generate mobility signaling inside the CSP domain.

\section{B. LISP: an inter-domain mobility solution}

Whereas PMIPv6 is well suited for intra-domain mobility, it cannot help managing mobile node inter-domain mobility. One solution is to use MIPv6 which is standardized in RFC 3775 [6] and is described for ATN/IPS internetwork in [7] and [2]. The main drawback of MIPv6 is that it requires a modification in the mobile node's IP stack to announce its Care-of-Address to the Home Agent, and thus implying additional signaling exchange in the radio link. Hence, a network-based solution is recommended such as LISP (or Ground LISP), which introduces a new paradigm to solve inter-domain mobility and multihoming.

LISP [8] defines a network based mobility solution allowing handoffs between different CSP domains and radio technologies. It splits the role of the IP address into 2 namespaces: Routing Locator (RLOC) and End-system Identifier (EID). RLOC are globally routable addresses used to localize the end-system and route the packet in the network, while EID are addresses dedicated to identify an end host. Doing so ensures a more flexible and scalable routing solution into the network. All the routing functionalities are moved to the ground with the Routing Locator Space (RLOC), and thus it does not require to make any modifications in the end-system, minimizing the implementation cost on-board. LISP routers, namely ingress Tunnel Router (iTR) and egress Tunnel Router (eTR), together 


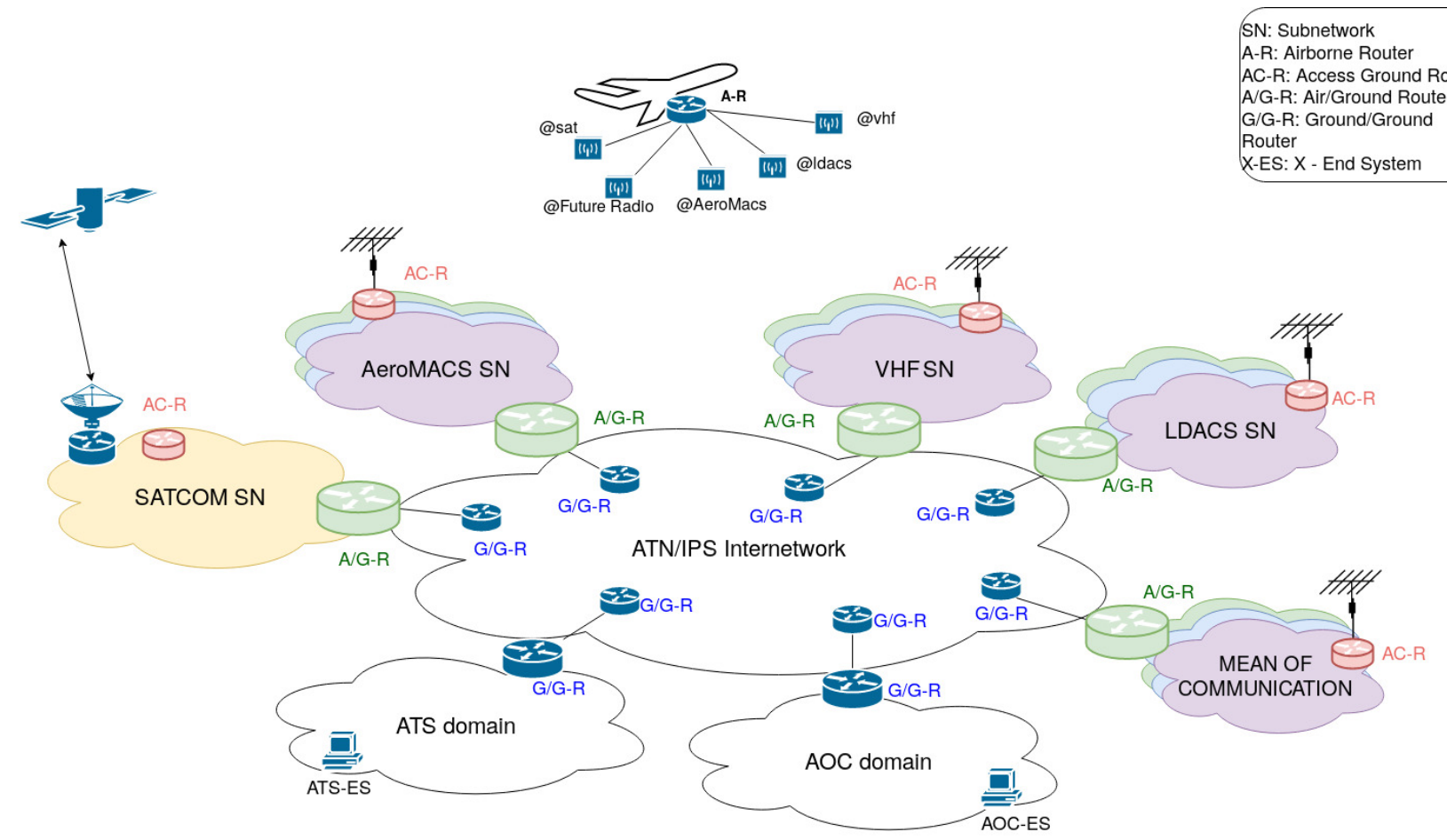

Fig. 1. The Future Communication Infrastructure

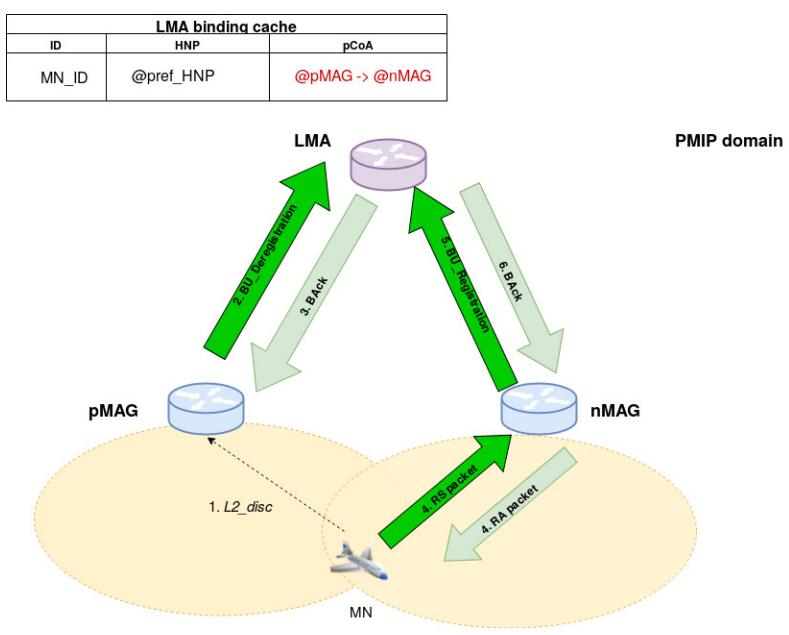

Fig. 2. PMIPv6 Handoff Management

with a mapping system maintain a mapping cache between the RLOC and the EID address, and use the encapsulation/decapsulation method to forward packets to the end-system. A detailed explanation of LISP for ATN/IPS can be found in [1], describing the different steps of the protocol. The interdomain handoff between 2 different CSPs in LISP is described in Figure 3. xTR refers to a LISP router implementing both iTR and eTR mechanisms. Once a MN leaves a CSP domain, its LISP eTR will inform the mapping system that the EID corresponding to the $\mathrm{MN}$ is no more reachable via its RLOC. When detecting a new MN in its domain, its LISP Router sends a MAP_Register message towards the mapping system to create a new EID-to-RLOC mapping for the MN.

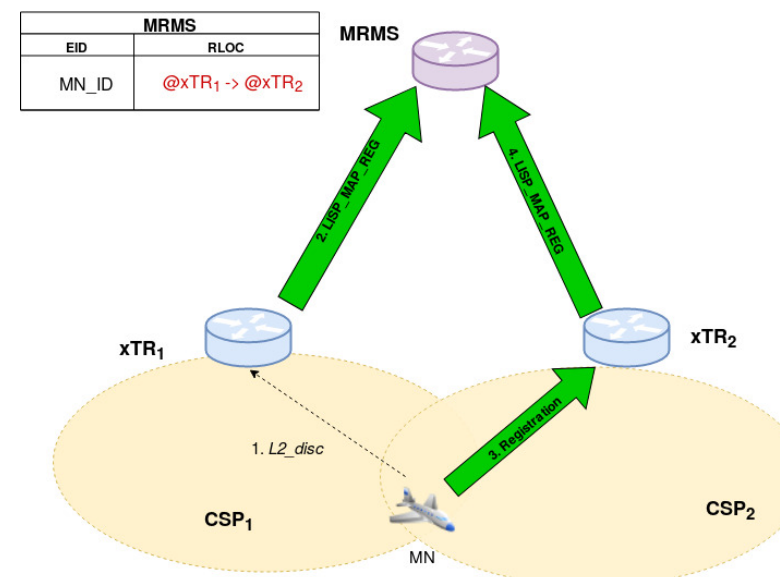

Fig. 3. LISP Handoff Management

\section{A new global mobility solution}

We previously described how the intra-domain and interdomain mobility should be resolved. Hence, we propose a solution integrating both protocols to manage the aircraft global mobility. The combination of these two approaches benefits from advantages of both sides, hence renders our solution more optimal. Figure 4 shows how this solution can be deployed in the FCI.

- Mapping System (MS/MR): it is an entity implemented in the ATN/IPS ground network. It is composed of 
a Map Server (MS) and a Map Resolver (MR). The MS is connected to the eTRs from which it receives LISP map regristration messages and stores the EID-toRLOC mapping into the database. Furthermore, it must advertises any new EID-to-RLOC mappings to iTRs that have already asked a mapping for this EID, in order to give them an up-to-date mapping information.

The MR responds to LISP map request messages sent by iTRs, whenever an EID-to-RLOC mapping needs to be performed. LISP protocol specifies 2 modes to forward EID-to-RLOC mapping, the direct and indirect mode, as described in [9]. Here, we choose to use the direct mode, where the MR directly replies to the iTR requesting the mapping, as it includes less signaling traffic.

- PMIP domain: it corresponds to a CSP domain, including a network access for each link technology type provided. However, a CSP providing a satellite link access may not be concerned. Indeed, the mobility inside this type of subnetwork may be solved with a L2 solution.

- AC-R: It takes the role of the MAG inside the PMIP domain. At least one AC-R per network access is recommended. It is responsible for handling the aircraft authentication in the ATN/IPS network and the aircraft mobility signaling. Whenever an aircraft detects a new network access and wants to establish a new connection, the AC-R will start the authentication procedure on behalf of the by querying the AAA server. After a correct authentication, the $\mathrm{AC}-\mathrm{R}$ will register the aircraft in the LMA.

- A/G-R: In our proposal, this router must integrate the role of both the LMA and the LISP router through eTR/iTR mechanism, and thus allowing the aircraft to be connected to the rest of the FCI. First, it will assign the aircraft home network prefix(HNP) based on ICAO IPS addressing space with 24 bits corresponding to the aircrafts ICAO ID. Then it will use this HNP as the aircraft EID and register it with its RLOC address (i.e its routable address belonging to the ATN/IPS domain) by sending a LISP_MAP_REGISTER message to the Mapping System (MS/MR).

In this way, ground end-systems willing to communicate with the aircrafts HNP will send, through their G/G router, a map request to the mapping resolver to get the corresponding RLOC address. Reversely, when an aircraft initiates the communication to a ground end-system, the A/G-R acts as an iTR, meaning that it has to send a map request to get the RLOC address attached to the ground end-system. After receiving a non empty LISP map reply, the A/G-R will store the mapping in its local cache and then create a LISP tunnel to the G/G-R having the corresponding RLOC address and send aircraft packets through this tunnel. In this way, for further incoming packets to forward, it just needs to use the corresponding tunnel.

- Ground/Ground-Router (G/G-R): It is a LISP router acting as an xTR, to interconnect the Applicative Service
Provider to the ATN/IPS core network. It is in charge of registering all the ground end-systems into the mapping system, and like the A/G-R, performing EID-to-RLOC mapping.

\section{Mobility scenario}

To highlight the mechanisms previously described, we consider the flight scenario in Figure 5 where the aircraft performs an inter-domain handoff from CSP1 to CSP2 keeping the same technology access. This type of handoff may happen when the first CSP does not cover the whole area with this technology access, thus requiring a need for an handoff to another CSP providing this type of access. Then, the aircraft remains in the area covered by CSP2 and realizes an intra-domain handoff, changing its network point of access.

1) Inter-domain handoff: Figure 6 shows an aircraft realizing an inter-domain handoff. When the aircraft detects a degradation in its communication, it sends a link_disc L2 message to the current AC-R2. The latter informs the A/G$\mathrm{R} 1$ that the aircraft leaves the access network with a Binding Deregistration message. The A/G-R1 must then send a LISP map register to the mapping system to erase the EID-to-RLOC mapping corresponding to its routing locator address. To prevent eTRs from keeping using the old mapping, the mapping system has to inform the concerned iTRs of this change. This could be realized with a publish/subscribe method in which the iTRs subscribe to the mapping systems update messages.

In parallel, the aircraft establishes the radio communication with the CSP2 access network, and sends a Router Solicitation packet to discover the access router on the link. After receiving the RS, the AC-R3 starts the PMIP registration by sending a proxy Binding Registration message to the A/G-R2 (authentication with a AAA server is omitted here). To get the aircraft $\mathrm{HNP}$, the A/G-R2 requests this information to the other A/G-Rs. Once it receives the reply, it sends back a Binding Acknowledgement message with the corresponding HNP to the AC-R3. In the end, the AC-R3 sends a Router Advertisement message to the aircraft advertising the same HNP from which it has already configured an IPv6 address. Hence, the aircraft does not have to configure a new IPv6 address and run the related duplicate address detection procedure.

In the same time, the A/G-R has to advertise the mapping system of a new EID-to-RLOC mapping with a LISP map register message, which in turn broadcasts this information to its subscribers, i.e the iTR that have a mapping with the corresponding aircraft EID.

2) Intra-domain handoff: The aircraft has entered the CSP2 domain and is attached to AC-R3. Figure 7 describes the messages exchange when the aircraft has to achieve an intradomain handoff in the CSP2 domain from AC-R3 to ACR4. With the use of PMIPv6 protocol, the signaling remains in the CSP2 domain and has no impact on the ATN/IPS ground internetwork, as the aircraft location has already been announced when it has previously entered the domain. Only the AC-Rs and the A/G-R are concerned by the mobility signaling. As described in III-A, the A/G-R2 updates its 


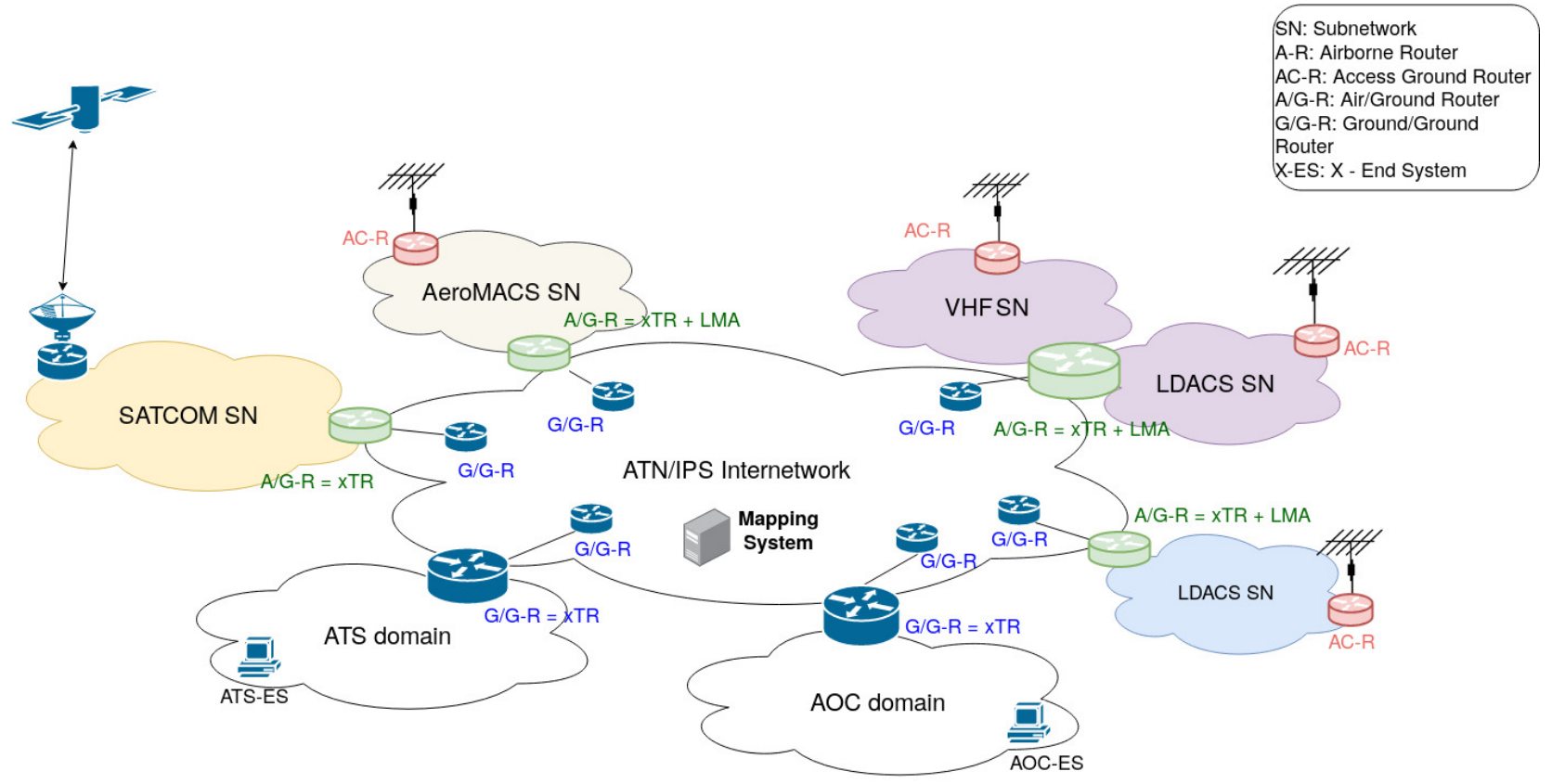

Fig. 4. Implementation of a global mobility solution in the FCI

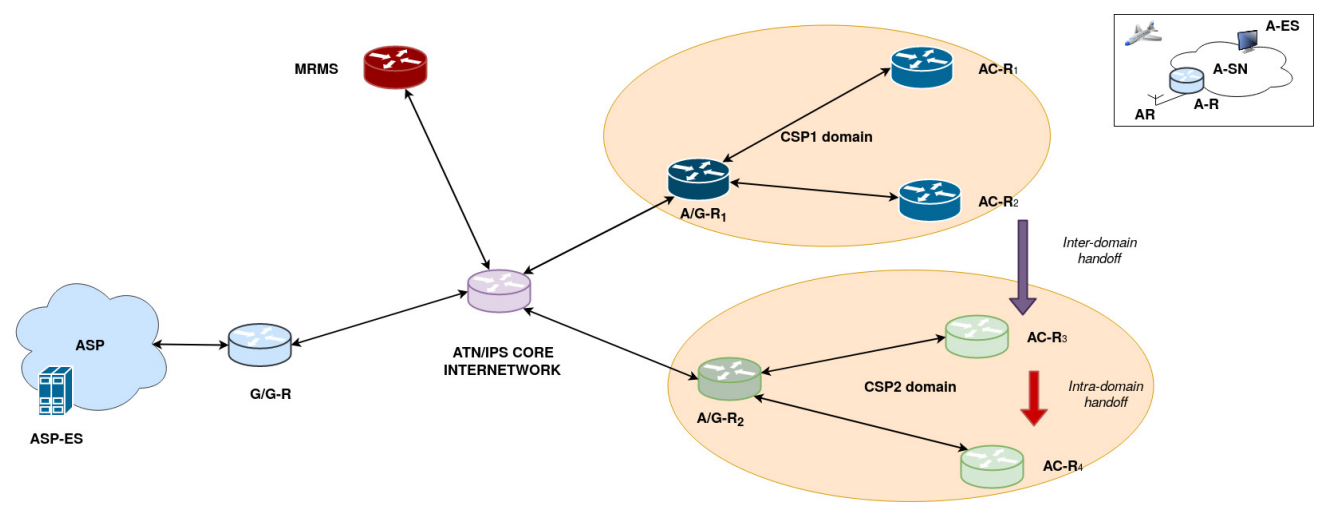

Fig. 5. Mobility scenario

binding cache entry concerning the location of the aircraft and sends back its HNP so it can continue to use the same IPv6 address.

\section{Performance Analysis and Evaluation}

Performance evaluation is based on the network infrastructure presented in Figure 5. Our solution is compared with the combination of MIPv6 with PMIPv6 to manage the aircraft global mobility, which is the first proposed solution to handle the global mobility with PMIPv6. Both solutions are implemented in the OMNeT++ software, based on the INET framework. The network infrastructure for the MIPv6/PMIPv6 solution is identical to our solution except that the Mapping system is replaced by the aircraft's Home Agent. In this scenario, the Home Agent attributes a home address to the aircraft, which is based on the ICAO address. When the aircraft is connected to a CSP, it is given a home network prefix, so that it can build a IPv6 Care-of-Address based on this prefix.
This paper focuses on the performances of the different handoffs at the network layer an aircraft has to perform during its trajectory. Hence, AC-Rs are equipped with a wireless network interface card to simulate air/ground datalinks and to communicate directly with the aircraft. However, to handle certain aspects of the layer 2 mechanism, a L2 manager was developed [4] and allows us to trigger a handoff at the layer 2 (when the signal is under a certain threshold for instance).

For both scenarios, we consider an aircraft moving at a cruise speed, performing successively an inter-domain handoff and an intra-domain handoff as described in the last part III-D. Meanwhile, it receives uplink trafic coming from the ASP endsystem. We implement a representative ATS and AOC traffic during an EN-ROUTE phase, proposed in [10], based on the UDP module of INET.

Figure 8 and Figure 9 show the uplink traffic end-to-end delay for both scenarios. They demonstrate that all the packets 


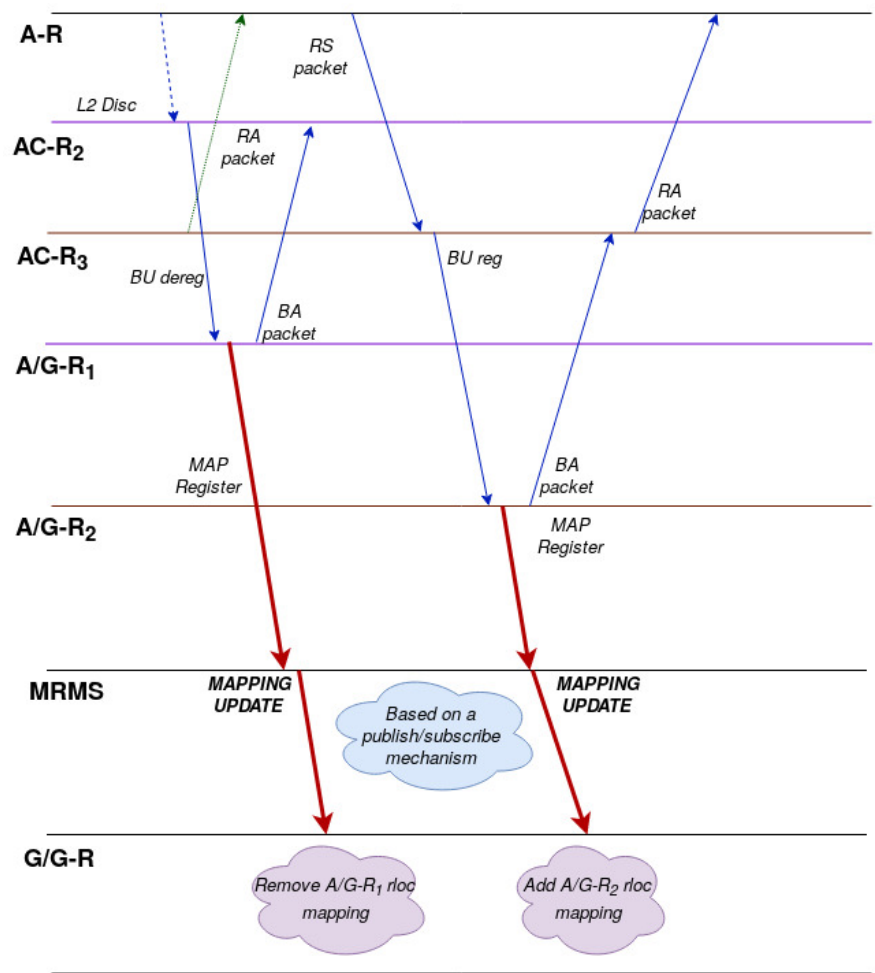

ASP-ES

Fig. 6. Inter-domain handoff mechanism

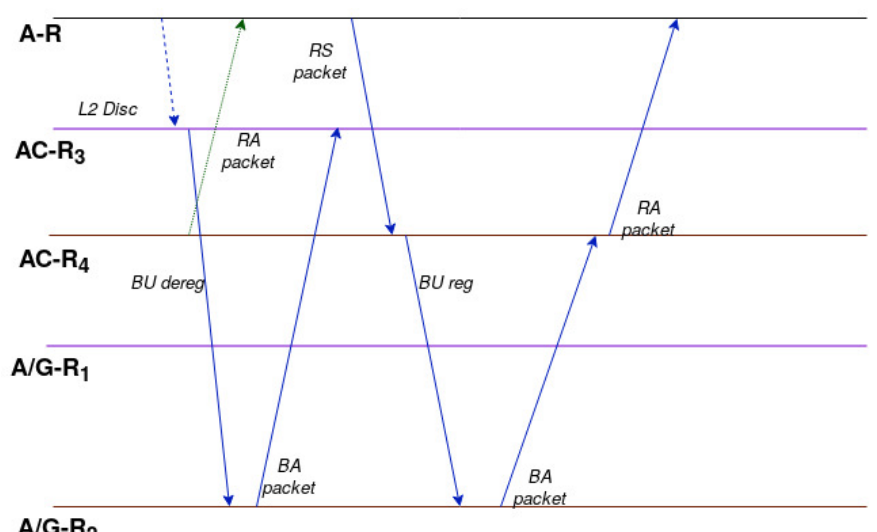

MRMS

G/G-R

ASP-ES

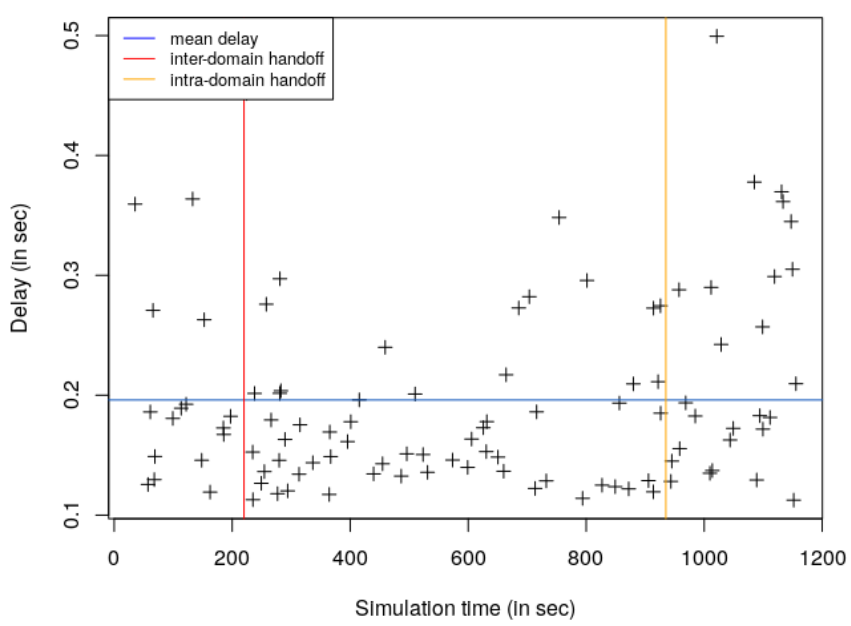

Fig. 8. Traffic application End-to-End delay for LISP scenario

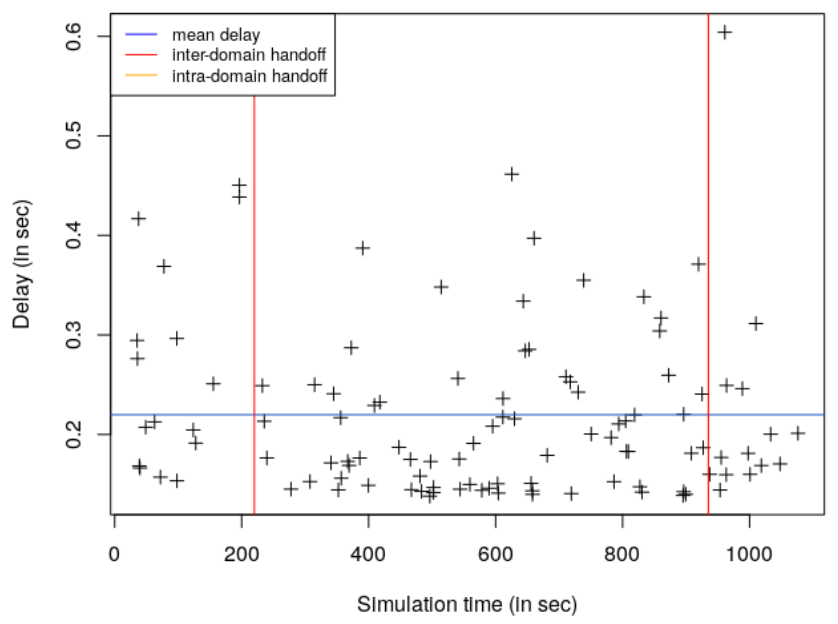

Fig. 9. Traffic application End-to-End delay for MIPv6 scenario

are indeed routed to the aircraft under an acceptable delay, even after the successive handoffs, happening at $t=220 \mathrm{~s}$ and $t=945 \mathrm{~s}$. Moreover, under these circumstances and hypotheses, our proposal with LISP is $10 \%$ more effective than the MIPv6 protocol in terms of average end-to-end delay. However, to further extend this result, other link technology types must be considered as we just model the case of an aircraft using VdLm2 subnetwork access.

We then evaluate both solutions in terms of handoff latency. The handoff delay for an intra-domain handoff depends only on the PMIPv6 protocol for both scenarios, so there is no difference between the two solutions. It corresponds to the delay from the moment when the aircraft loses its connection with the previous access router until the moment when the 
A/G-R updates its binding cache entry to insert the new aircraft's proxy Care-of-Address (pCoA). We define the interdomain handoff delay as the delay from the L2 disconnect signal emitted by the aircraft until the time it takes to the G/G$\mathrm{R}$ to update its EID-to-RLOC mapping for the LISP scenario, and until the time it takes to the Home Agent to get the new aircraft's Care-of-Address for the second scenario. This handoff delay is based on 3 factors:

1) New link detection: it corresponds to the time T1 until the aircraft get a new available link and emits a RS message through this link. This time is mainly due to the air/ground datalink protocols (like LDACS) and is not dependent on the network mobility solutions. In our simulation, we do not implement such protocols. Nevertheless, we have a L2 switching mechanism which simulates the same behavior and allows the on-board radio to be "connected" to a unique A/G datalink subnetwork.

2) Local update location: For LISP, it corresponds to the time period $\mathrm{T} 2$ from the new link detection until the xTR router gets the new location of the aircraft, i.e when it receives the Binding Registration from an AC-R. For MIPv6, it corresponds to the time period T2 until the aircraft gets its new IPv6 address from the new home network prefix it receives. It includes the duplicate address detection period the aircraft has to perform in order to validate the new IPv6 address.

3) Cache update: it is the time period T3 until the information of the new location of the aircraft reaches the router responsible to route the packet towards the aircraft. This router is the G/G-R for the LISP protocol, whereas it corresponds to the Home Agent for the MIPv6 protocol.

Figure 10 and 11 show the complete inter-domain handoff procedure for both cases. As said earlier, the handoff delay depends on the link technology type. To provide realistic results, we model the delay induced by the MAC sublayer before sending the packets. This delay is representative of a delay perceived by an aircraft in a VHF Datalink Mode 2 (VDLm2) cell (one of the link technology types in ATN/IPS). This study is performed with the VDLm2 protocol developed under OMNeT++ at the ENAC laboratory. We show that the time in queue for downlink and uplink packets follow an exponential law with $\lambda$ depending on the number of aircraft in the cell. The following results are obtained by varying the cell capacity from 20 to 200 aircraft.

The time $\mathrm{T} 1$ is identical for both case scenario as it does not depend on any network layer mechanism. For the LISP solution, we do not take into account the time period until the aircraft receives the HNP inside the solicited RA message as it will receive the same HNP. The time T2 is greater for the MIPv6 scenario because of the DAD procedure which takes approximately 2 seconds, 1 second to join the multicast group, and 1 second to be sure that no other nodes on the link is using the same address. Concerning the time period T3, it is much shorter with our solution as the mobility signaling remains in the network, unlike the MIPv6 scenario where the signaling involves the aircraft. Overall, with our solution, it takes $3,11 \mathrm{~s}$ at maximum to perform the inter-domain handoff meanwhile with the MIPv6 protocol, the inter-domain handoff delay can reach $6,59 \mathrm{~s}$. This result proves that using LISP can help to reduce the handoff delay by 2 .

\section{CONCLUSION}

Coupling LISP with PMIPv6 is a promising solution. It is a full network-based mobility solution keeping on-board complexity as low as possible. Compared to a solution involving MIPv6 protocol for inter-domain mobility where the aircraft is in charge of announcing its location to the Home Agent, it minimizes the mobility signaling exchange with the aircraft, thus saving radio resources. We provide an $\mathrm{OMNeT}++$ simulation model of both LISP and MIPv6 protocols combined with PMIPv6 and we evaluate the performances of successive handoffs. Firstly, the application data end-to-end delay proves that our solution can efficiently manage the different handoff case scenarios, and performs slightly better than the MIPv6 based solution. Results also show that the LISP based solution performs inter-domain handoff faster than the MIPv6 based solution.

To further extend our study, we will implement a modelisation of other link technology types foreseen for ATN/IPS (like LDACS and SATCOM) and verify whether we obtain the same results as shown in this paper. Also, the multilink capability, which is identified as a key aspect to provide high availability and quality in the FCI, of this protocol will be investigated later on. 


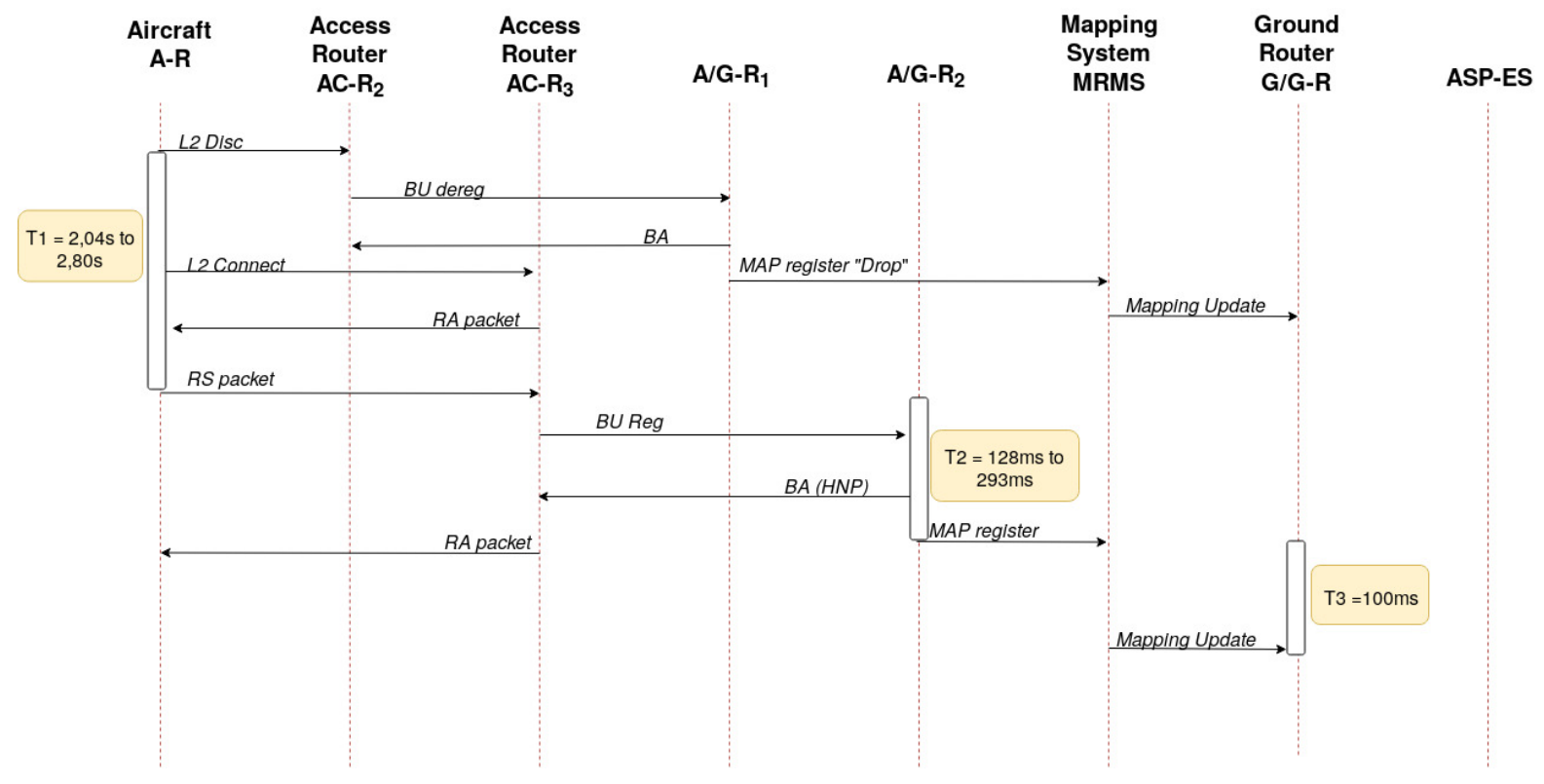

Fig. 10. Inter-domain handoff delay with LISP

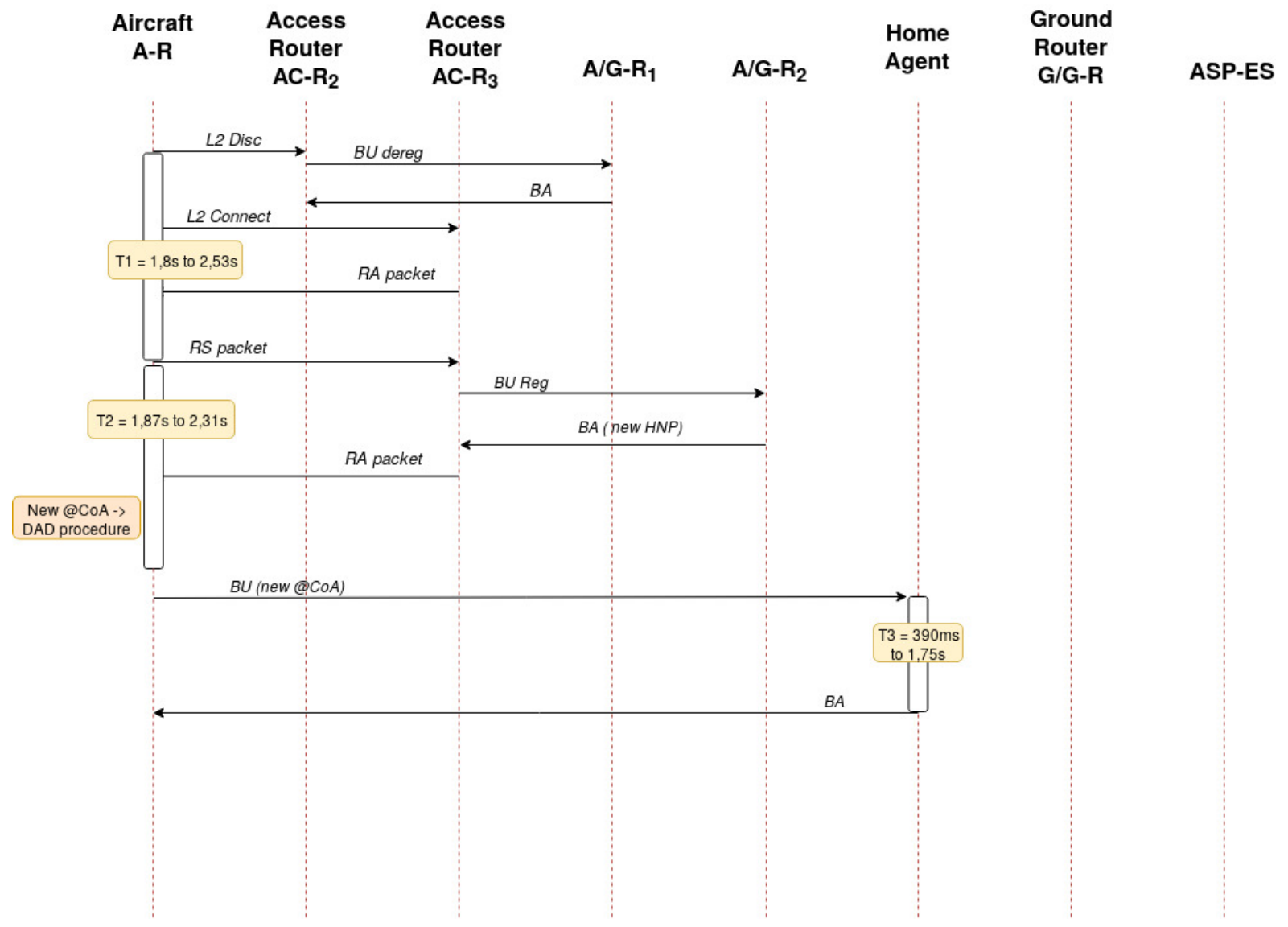

Fig. 11. Inter-domain handoff with MIPv6 


\section{REFERENCES}

[1] B. Haindl and M. Lindner, "Ground based lisp for multilink operation in atn/ips communication infrastructure," 09 2016, pp. 1-10.

[2] T. McParland, "Multihoming in the atn/ips," in 2016 Integrated Communications Navigation and Surveillance (ICNS), April 2016, pp. 2B2$1-2 \mathrm{~B} 2-11$.

[3] F. Templin, "Asymmetric Extended Route Optimization (AERO)," Internet Engineering Task Force, Internet-Draft draft-templin-intarea6706bis-12, May 2019, work in Progress. [Online]. Available: https://datatracker.ietf.org/doc/html/draft-templin-intarea-6706bis-12

[4] A. Tran, A. Pirovano, N. Larrieu, A. Brossard, and S. Pelleschi, "IP Mobility in Aeronautical Communications," in Nets4, 13th International Workshop on Communication Technologies for Vehicles, Nets4Aircraft and UAV session, ser. Communication Technologies for Vehicles - 13th International Workshop, Nets4Cars/Nets4Trains/Nets4Aircraft 2018, vol. Lecture Notes in Computer Science, no. 10796,. Madrid, Spain: Springer, May 2018, pp. pp 16-26 / ISBN 978-3-319-90 370-5. [Online]. Available: https://hal-enac.archives-ouvertes.fr/hal-01785047

[5] K. Chowdhury, K. Leung, B. Patil, V. Devarapalli, and S. Gundavelli, "Proxy Mobile IPv6," RFC 5213, Aug. 2008. [Online]. Available: https://rfc-editor.org/rfc/rfc5213.txt

[6] C. E. Perkins, J. Arkko, and D. B. Johnson, "Mobility Support in IPv6," RFC 3775, Jun. 2004. [Online]. Available: https://rfceditor.org/rfc/rfc3775.txt

[7] C. Bauer and S. Ayaz, "A thorough investigation of mobile ipv6 for the aeronautical environment," in 2008 IEEE 68th Vehicular Technology Conference, Sep. 2008, pp. 1-5.

[8] D. Farinacci, V. Fuller, D. Meyer, and D. Lewis, "The Locator/ID Separation Protocol (LISP)," RFC 6830, Jan. 2013. [Online]. Available: https://rfc-editor.org/rfc/rfc6830.txt

[9] C. Caiazza, A. Virdis, and G. Stea, "Simulating lisp-based multilink communications in aeronautical networks," in Proceedings of the 5th International OMNeT++ Community Summit, ser. EPiC Series in Computing, A. F "orster, A. Udugama, A. Virdis, and G. Nardini, Eds., vol. 56. EasyChair, 2018, pp. 43-51. [Online]. Available: https://easychair.org/publications/paper/KCXR

[10] L. Z. Ribeiro, L. C. Monticone, R. E. Snow, F. Box, R. Apaza, and S. Bretmersky, "A framework for dimensioning vdl-2 air-ground networks," in 2014 Integrated Communications, Navigation and Surveillance Conference (ICNS) Conference Proceedings, April 2014, pp. Q31-Q3-14. 